\section{Bilateral foot drop following lower limb orthopedic surgery under spinal anes- thesia}

To the Editor:

Neurological complications, though rare, have been documented after spinal anesthesia. The exact etiological factor may not be detected in all patients. Traumatic injury to the spinal cord or nerve roots during spinal anesthesia is an infrequent cause. ${ }^{1}$

A 35-yr-old male trauma patient with a fracture of the right femur and compound fractures of both legs was scheduled for open reduction and internal fixation of the right femur. Tibial pins had been inserted under local anesthesia. Preoperative investigations and vital signs were within normal limits. Spinal anesthesia was administered using a $25-\mathrm{G}$ Whitacre needle and $2.6 \mathrm{~mL}$ of $0.5 \%$ hyperbaric bupivacaine at the L3 to L4 interspace. The patient was found to have bilateral foot drop on the second postoperative day. Dorsiflexion had not been tested preoperatively, and he was unaware of its existence as well. The neurologist recommended electromyography (EMG) and magnetic resonance imaging (MRI) of the spine. The EMG did not reveal intradural pathology. The neurological examination showed normal sensation on the exposed aspects of both thighs and legs except for numbness on the dorsal aspect of right first toe. Ankle and knee reflexes could not be elicited due to bandaging and splinting of the limbs. An MRI scan of the spine showed normal spinal anatomy.

Neurological complications due to spinal anesthesia must be suspected in any patient developing neurological signs or symptoms within eight months after anesthesia. ${ }^{2}$ Incidence of nerve injury related to spinal anesthesia is less than $1: 10,000 .{ }^{3}$ Foot drop can occur due to paralysis of foot flexors (sciatic or common peroneal nerve injury). If an injury is expected at a higher level, then careful testing of the thigh and hip muscles must be performed for localization. There is diffuse muscle involvement and an absent ankle reflex after a central injury. In our patient, localization was limited due to splinting. Needle trauma due to multiple attempts is a rare cause, however, all traumatic lumbar punctures may not be followed by neurological complaints. Paresthesias during lumbar puncture may correlate with neurological sequela but pain should also be present during the injection of a drug.

Reynolds has reported foot drop in six patients, all of whom revealed unilateral neurological deficit and MRI findings of unilateral damage to the conus medullaris. ${ }^{4}$ In contrast, the MRI scan of our patient was normal, ruling out injury to the cauda. Moreover, the EMG also ruled out intradural pathology. Foot drop has also been reported after passage of steinman pin postoperative during femur surgery, and due to nerve avulsion in compound fractures. ${ }^{5}$ Bilateral lesions after spinal anesthesia are possible only if a lumbar puncture is performed above the second interspace. Even after correct identification of interspaces, dural punctures have a chance of being performed in spaces higher than assumed 'anding to possible unilateral lesions of the conus.

We recommend a limited neurological examination of the lower limbs prior to spinal anesthesia.

Anju Ghai MD

Sarla Hooda MD

Prashant Kumar MD

Raman Kumar MD

Pankaj Bansal MD

Sharma Post Graduate Institute of Medical Sciences, Haryana, India

E-mail: prashantkumarz@yahoo.com

\section{References}

1 Auroy $\Upsilon$, Benhamou D, Bargues L, et al. Major complications of regional anesthesia in France: The SOS Regional Anesthesia Hotline Service. Anesthesiology 2002; 97: 1274-80.

2 Kane RE. Neurologic deficits following epidural or spinal anesthesia. Anesth Analg 1981; 60: 150-61.

3 Bajaj P. Complications of central neuraxial blockade. Indian J Anaesth 2001; 45: 445-55.

4 Reynolds F. Damage to the conus medullaris following spinal anaesthesia. Anaesthesia 2001; 56: 238-47.

5 John Ebnezar. Peripheral nerve injury. In: (Ed.). Textbook of Orthopaedics, 2nd ed. Philadelphia: Jay Pee Brothers; 2000: 168-80.

\section{Endobronchial metastasis: an anesthet- ic complication?}

To the Editor:

A 55-yr-old man presented with dyspnea and hemoptysis six months after completion of external radiation to the head and neck. Ten months previously he had undergone radical surgery for carcinoma tongue (T4N0M0). Review of his previous records revealed that he had required an awake fibreoptic intubation. Considerable difficulty was encountered in negotiating the tracheal tube due to posterior extension of the tumour. The intraoperative and postoperative periods were uneventful and he made satisfactory recovery. The 